\title{
The Complex X-ray Spectra of M82 and NGC 253
}

\author{
A. Ptak ${ }^{1}$, P. Serlemitsos, T. Yaqoob ${ }^{2}$, R. Mushotzky \\ NASA/Goddard Space Flight Center, Code 662, Greenbelt, MD 20771, USA \\ and \\ T. Tsuru \\ Department of Physics, Faculty of Science, Kyoto University, Sakyo-ku, Kyoto 606-01, Japan
}

\begin{abstract}
We present the results of the first imaging X-ray observations in the 0.4-10.0 keV bandpass of the nearby starburst galaxies M82 and NGC 253. The ASCA spectra of both M82 and NGC 253 are complex with strong line emission from $\mathrm{O}, \mathrm{Ne}, \mathrm{Fe}, \mathrm{Mg}, \mathrm{S}$, and $\mathrm{Si}$, allowing elemental abundances to be estimated in the X-ray band for the first time in these sources. Two components are required to fit the spectra of both galaxies, with a "soft" component well described by a thermal model with a temperature of $\sim 10^{6-7} \mathrm{~K}$ and a "hard" component well described by either a thermal model $\left(T_{\text {hard }} \sim 10^{8} \mathrm{~K}\right)$ or a power-law model $(\Gamma \sim 1.8-2.0)$. We find that different models (with different continua) yield absolute abundances that differ by more than an order of magnitude, while relative abundances are more robust and suggest an underabundance of Fe (inferred from the Fe-L complex) relative to $\alpha$-burning elements. Dust depletion may be responsible for the low relative abundance of Fe inferred from the soft-component fits. We set the most reliable limits to date on the Fe-K emission line equivalent width (EW), with an upper limit in NGC 253 of $\mathrm{EW}<180 \mathrm{eV}$ while a line at $\sim 6.6 \mathrm{keV}$ is marginally
\end{abstract}

\footnotetext{
${ }^{1}$ University of Maryland, College Park, MD 20742, USA

${ }^{2}$ With the Universities Space Research Association
} 
significant (at the $3 \sigma$ level) with $\mathrm{EW} \sim 100 \mathrm{eV}$ in M82. The low Fe-K line emission EW limits suggest either significantly sub-solar abundances in the material producing the hard component (if thermal) or that there is a significant non-thermal or non-equilibrium contribution to the hard component. Most of the soft flux (which originates mostly within the central kpc of M82 and NGC 253) is consistent with starburst models of supernovae-heated ISM and, to a lesser extent, starburst-driven superwind emission and the direct emission from supernova (SN). The hard component in both galaxies may have some contribution from $\sim 10^{8}$ $\mathrm{K}$ superwind emission or individual SN, although most of the emission probably originates in point sources (most likely blackhole candidates or mini-AGN with $M_{E d d}>3-20 M_{\odot}$ ) and, possibly, inverse-Compton scattering of IR photons. The similarity of the spectral X-ray characteristics of NGC 253 and M82 to some LINERs and low-luminosity AGN suggests a link between AGN and starbursts (e.g., both may contain an accretion-driven emission component).

Subject headings: galaxies: starburst - galaxies: abundances - galaxies: individual (NGC 253, M82) - X-rays: galaxies 


\section{Introduction}

M82 and NGC 253 are two of the nearest "starburst" galaxies (we assume distances of 3.6 and $2.5 \mathrm{Mpc}$, respectively; Freedman et al. 1994; Davidge \& Prichett 1990) where extensive star formation in the $<1$ kpc-scale nuclear regions (Rieke et al. 1980) has resulted in a spectacular X-ray morphology, with emission concentrated both in the nucleus (at levels of one to two orders of magnitudes higher than typically seen in normal spirals) and extended along their minor axes to scales of up to $\sim 10 \mathrm{kpc}$. M82 and NGC 253 have been extensively studied in X-rays, with data from the Einstein HRI (Watson, Stanger, \& Griffiths 1984; Fabbiano \& Trinchieri 1984), ROSAT HRI (Bregman, Schulman, \& Tomisaka 1995) and EXOSAT LE (Schaaf et al. 1989) revealing the X-ray morphology below $\sim 4 \mathrm{keV}$, showing that much of the nuclear emission is concentrated in multiple point sources. Data from the Einstein IPC (Fabbiano 1988), EXOSAT ME (Schaaf et al. 1989), Ginga (Ohashi et al. 1990; Tsuru et al. 1990) and BBXRT (Petre 1994) suggest a complex spectrum with at least two components, although the statistics, spectral resolution, and/or bandpass were somewhat limited with these instruments. These studies have suggested that the nuclear Xray emission is due to an extended component probably consisting of the emission of unresolved supernovae (SN) and SN-heated ISM, and individual point sources which are likely to be very luminous X-ray binaries and young supernova (SN) or possibly even a hidden AGN. These studies also suggest that the "plumes" breaking out of their galactic disks are evidence for SN-driven "superwinds" (Heckman, Armus \& Miley 1990) based on the observed temperatures and luminosi- ties. Inverse-Compton (IC) scattering of the large IR flux $\left(L_{I R} \sim 10^{10} L_{\odot}\right.$; Telesco 1988) by relativistic electrons has also been suggested as a significant contributor to the X-ray flux (Rieke et al. 1980; Schaaf et al. 1989; Moran \& Lehnert 1996), a view (possibly) supported by the detection of $\mathrm{MeV}$ emission in the vicinity of NGC 253 (Bhattacharya et al. 1994). However, the expected intensity of the IC component is subject to large uncertainties depending on model assumptions (see $\S 4$ ).

The significant spatial and spectral complexity suggests that proper deconvolution of the spectra would require good spatial and spectral resolution. Here we present highquality spectra of M82 and NGC 253 in the 0.4-10.0 keV bandpass obtained by the X-ray satellite $A S C A$ which carries the first imaging, medium-resolution X-ray spectrometers. Although the spatial resolution of $A S C A$ is inferior to that of ROSAT, ASCA can constrain the source of the X-ray emission to within $\sim 1^{\prime}$, while previous detectors with significant effective area above $2 \mathrm{keV}$ were essentially non-imaging (although BBXRT allowed for some crude spatial analysis). We show below that the spectra are indeed complex, showing strong line emission and requiring multiple components.

\section{The ASCA Data}

M82 was observed by $A S C A$ (Tanaka, Inoue, \& Holt 1994) on May 19, 1993 and NGC 253 was observed on June 10, 1993. Briefly, $A S C A$ consists of two solid-state imaging spectrometers (SIS; hereafter S0 and S1) with an approximate bandpass of $0.4-10.0 \mathrm{keV}$ and two gas imaging spectrometers (GIS; hereafter S2 and S3) with an approximate bandpass of $0.8-10.0 \mathrm{keV}$. The SIS observations were done in 4-ccd mode although only the 
data from S0 chips 1 and 2 and S1 chips 0 and 3 (containing most of the source counts) were used in the present analysis. Times of high background were excluded and hot pixels (in the SIS data) were removed. In M82 and NGC 253, most of the extended emission is concentrated within $\sim 1$ ' of the nucleus (Fabbiano \& Trinchieri 1984; Watson, Stanger \& Griffiths 1984; Bregman, Schulman, \& Tomisaka 1995). In NGC 253, several point sources are clearly present at distances of up to several arcminutes from the nucleus (see below). Accordingly, source counts were extracted from a 6' radius circle centered on the nuclear source in each instrument (source regions sizes of 3' and 4' are typically chosen for the SIS and GIS, respectively, in the case of a single point source observation). The background was determined from the remaining counts in the chips beyond 6.' 5 for the SIS, from a 8-13' annulus for M82 GIS data and from an offset 5' circle for the NGC 253 GIS data. The observed M82 count rates were 0.61.0 counts $\mathrm{s}^{-1}$ (per detector) among the four detectors with net exposure times of $\sim 17$ $20 \mathrm{ks}$ and the background comprising 4-6\% of the total. The observed NGC 253 count rates were $0.1-0.3$ counts $\mathrm{s}^{-1}$ among the four detectors with net exposure times of $\sim 27-30$ ks and the background comprising $12-19 \%$ of the total.

ROSAT PSPC images of M82 and NGC 253 show that their extended halos are soft (i.e., $\mathrm{kT} \lesssim 0.5 \mathrm{keV}$ ), contributing negligibly in the $\sim 0.5-2.0 \mathrm{keV}$ bandpass compared to the X-ray flux originating within the 6' source regions described above. Nevertheless, it is possible that the background regions used in extracting the $A S C A$ background spectra may be contaminated by the scattering of source photons or, possibly, some (relatively hard) non-thermal emission associated with the radio synchrotron halos observed in the halos of M82 and NGC 253 (c.f., Seaquist \& Odegard 1991; Carilli et al. 1992). The GIS spectra are particularly vulnerable to these effects given the poorer spatial resolution and higher sensitivity above $2 \mathrm{keV}$ of the GIS relative to the SIS. However, we find that the counting rate in our background regions are consistent with spectra extracted from blank sky fields. Substituting the blank sky background spectra for the background spectra described above does not significantly affect any of the spectral results discussed below. The telescope response was calculated assuming most of the counts within 6 ' are consistent with a point source (i.e., a source extent of $<$ $\left.1^{\prime}\right)$. We went to some considerable effort in investigating the effect of neglecting the spatial complexity on the spectral analysis and these results are described in the appendix. We find that neglecting the spatial complexity does not have a siginificant impact on our results. The spectra were binned to a minimum of 10 counts per bin to allow use of the $\chi^{2}$ statistic.

\section{Spectral Fitting}

The SIS spectra strongly rule out a singlecomponent model from 0.4-10.0 keV, with both power-law and Raymond-Smith plasma fits resulting in $\chi_{\nu}^{2}>2.0$ for both sources. The poorer spectral resolution and response (at low energies) of the GIS allow for a singlecomponent model. Indeed, most of the contribution to $\chi^{2}$ in the SIS fits results from narrow features below $\sim 2.5 \mathrm{keV}$. This can be seen in Figure 1 which shows the ratio of data to best-fitting power-law model, with the location of expected $\mathrm{K}_{\alpha}$ line emission from the He- and H-like ions of $\mathrm{O}, \mathrm{Ne}, \mathrm{Mg}$, Si, 
TABLE 1

Two-component Model Fits to ASCA M82 and NGC 253 Spectra

\begin{tabular}{cccccccc}
\hline \hline Galaxy/Detector & Model $^{*}$ & $N_{H}^{\text {soft }}$ & $\mathrm{kT}(\mathrm{keV})$ & $N_{H}^{\text {hard }}$ & $\Gamma / \mathrm{kT}(\mathrm{keV})$ & $A / A_{\odot}^{\dagger}$ & $\chi^{2} / \mathrm{dof}$ \\
\hline N253/S0-1 & 1 & $1.0_{-1.0}^{+0.7}$ & $0.81_{-0.19}^{+0.06}$ & $15_{-11}^{+15}$ & $2.02_{-0.29}^{+0.51}$ & & $416.9 / 360$ \\
$\mathrm{~N} 253 / \mathrm{S} 0-3$ & 1 & $1.0_{-1.0}^{+0.8}$ & $0.80_{-0.18}^{+0.06}$ & $14_{-10}^{+13}$ & $2.01_{-0.40}^{+0.40}$ & & $915.0 / 870$ \\
$\mathrm{~N} 253 / \mathrm{S} 0-1$ & 2 & $0.8_{-0.8}^{+0.9}$ & $0.81_{-0.16}^{+0.06}$ & $8_{-8}^{+13}$ & $7.2_{-3.2}^{+9.8}$ & $0.03_{-0.03}^{+0.32}$ & $423.7 / 359$ \\
$\mathrm{~N} 253 / \mathrm{S} 0-3$ & 2 & $0.8_{-0.8}^{+0.9}$ & $0.81_{-0.14}^{+0.06}$ & $8_{-8}^{+11}$ & $7.3_{-2.7}^{+5.6}$ & $0.02_{-0.02}^{+0.23}$ & $922.3 / 869$ \\
$\mathrm{M} 82 / \mathrm{S} 0-1^{\ddagger}$ & 1 & $2.4_{-0.8}^{+0.7}$ & $0.64_{-0.32}^{+0.18}$ & $19_{-19}^{+12}$ & $1.78_{-0.47}^{+0.35}$ & & $548.1 / 422$ \\
$\mathrm{M} 82 / \mathrm{S} 0-3$ & 1 & $2.4_{-0.6}^{+0.7}$ & $0.63_{-0.30}^{+0.13}$ & $19_{-10}^{+10}$ & $1.76_{-0.21}^{+0.22}$ & & $1379 / 1334$ \\
$\mathrm{M} 82 / \mathrm{S} 0-1$ & 2 & $2.3_{-2.3}^{+0.7}$ & $0.63_{-0.33}^{+0.15}$ & $14_{-14}^{+11}$ & $10_{-4 .}^{+45}$ & $0.19_{-0.19}^{+0.42}$ & $540.7 / 421$ \\
$\mathrm{M} 82 / \mathrm{S} 0-3^{\ddagger}$ & 2 & $2.3_{-0.8}^{+0.7}$ & $0.63_{-0.32}^{+0.13}$ & $14_{-14}^{+10}$ & $11.4_{-4.4}^{+9.3}$ & $0.18_{-0.18}^{+0.35}$ & $1366 / 1333$ \\
\hline
\end{tabular}

Note. - The errors give the $90 \%$ confidence intervals assuming that all model parameters (except for normalizations) are interesting. Soft-component abundances derived from the 4-instrument Raymond-Smith plus power law fits are given in text.

* 1: Raymond-Smith plus power law, 2: Raymond-Smith plus Raymond-Smith

$\dagger$ Hard-component abundance relative to solar

$\ddagger$ Due to a relative minimum in $\chi^{2}$ space, the true soft component absoption column lower confidence limit may be zero (see text).

$N_{H}^{s o f t}$ is in units of $10^{21} \mathrm{~cm}^{-2}$, applied to both continuum components

$N_{H}^{\text {hard }}$ is in units of $10^{21} \mathrm{~cm}^{-2}$, applied to hard continuum component only 
$\mathrm{S}, \mathrm{Ca}$ and $\mathrm{Fe}$ and the L-shell emission of $\mathrm{Fe}$ marked. The prominence of He-like $\mathrm{Mg}$, Si and $\mathrm{S}$ lines implies a thermal component with kT $<2$ keV (c.f., Mewe, Gronenschild, \& Van Den Oord 1985). Accordingly we tried fitting two-component models to the spectra, with a Raymond-Smith model to account for the soft $(\lesssim 2 \mathrm{keV}$ ) flux and a Raymond-Smith or power-law component for the hard $(\gtrsim 2 \mathrm{keV})$ flux. These models resulted in acceptable fits for both NGC 253 and M82, although the SISonly M82 fits resulted in $\chi_{\nu}^{2} \sim 1.3$ which can be rejected at a level of $>99 \%$ (note that this neglects any systematic error).

The results are shown in Table 1, where fits to the SIS data alone are denoted 'S01 ' and fits to the data from all four instruments are denoted 'S0-3'. Table 1 shows that the S0-1 and S0-3 fits give consistent results. The errors given in Table 1 (and in the text below) assume that all model parameters are interesting except for normalizations, or 12 (8) interesting parameters for the M82 (NGC 253) Raymond-Smith plus power-law fits and 13 (9) interesting parameters for the double Raymond-Smith fits (the M82 and NGC 253 fits have a different number of model parameters due to a difference in the handling of the soft-component abundances, as discussed below). The double Raymond-Smith model gives a better fit to the M82 spectra while the Raymond-Smith plus power-law model gives a better fit to the NGC 253 spectra, although the differences in $\chi^{2}$ between the double Raymond-Smith and Raymond-Smith plus power-law models are statistically insignificant. The hard and soft components appear to be absorbed well in excess of the Galactic columns $\left(1.6 \times 10^{20} \mathrm{~cm}^{-2}\right.$ and $4.3 \times 10^{20} \mathrm{~cm}^{-2}$ for NGC 253 and M82, respectively; Stark et al. 1992), although

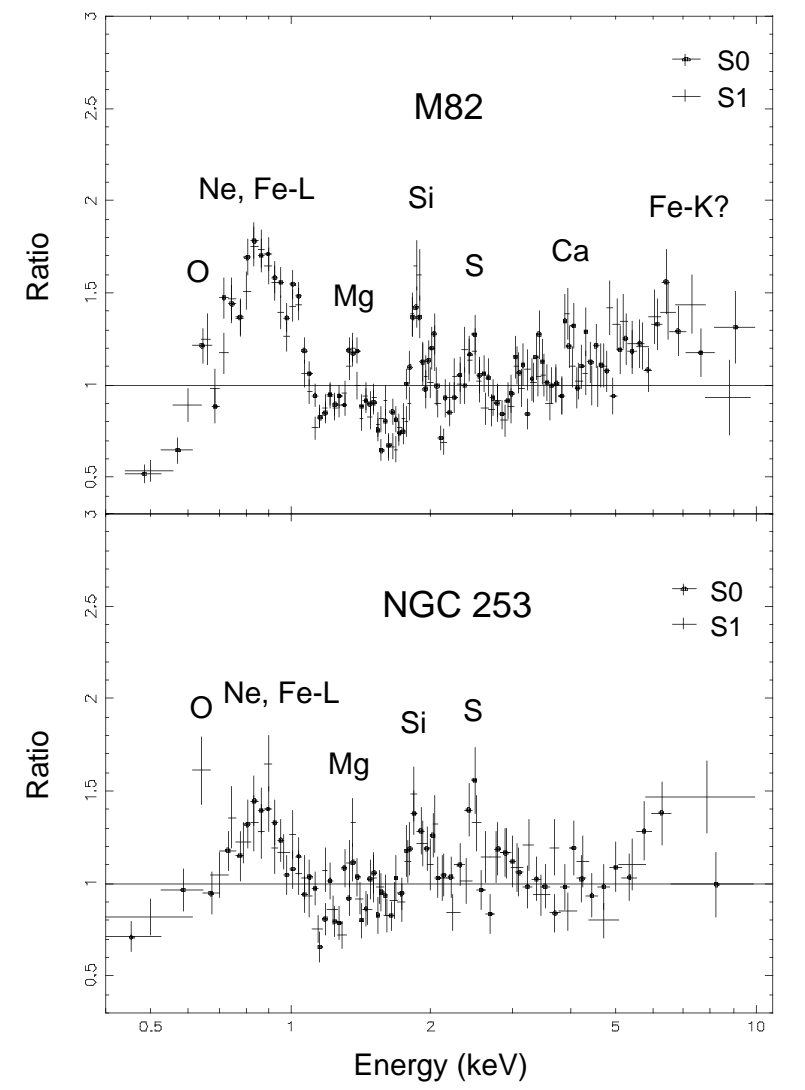

Fig. 1.- Ratio of the SIS $A S C A$ data to bestfitting power-law model for M82 (top) and NGC 253 (bottom). The possible identification of prominent lines is shown. 


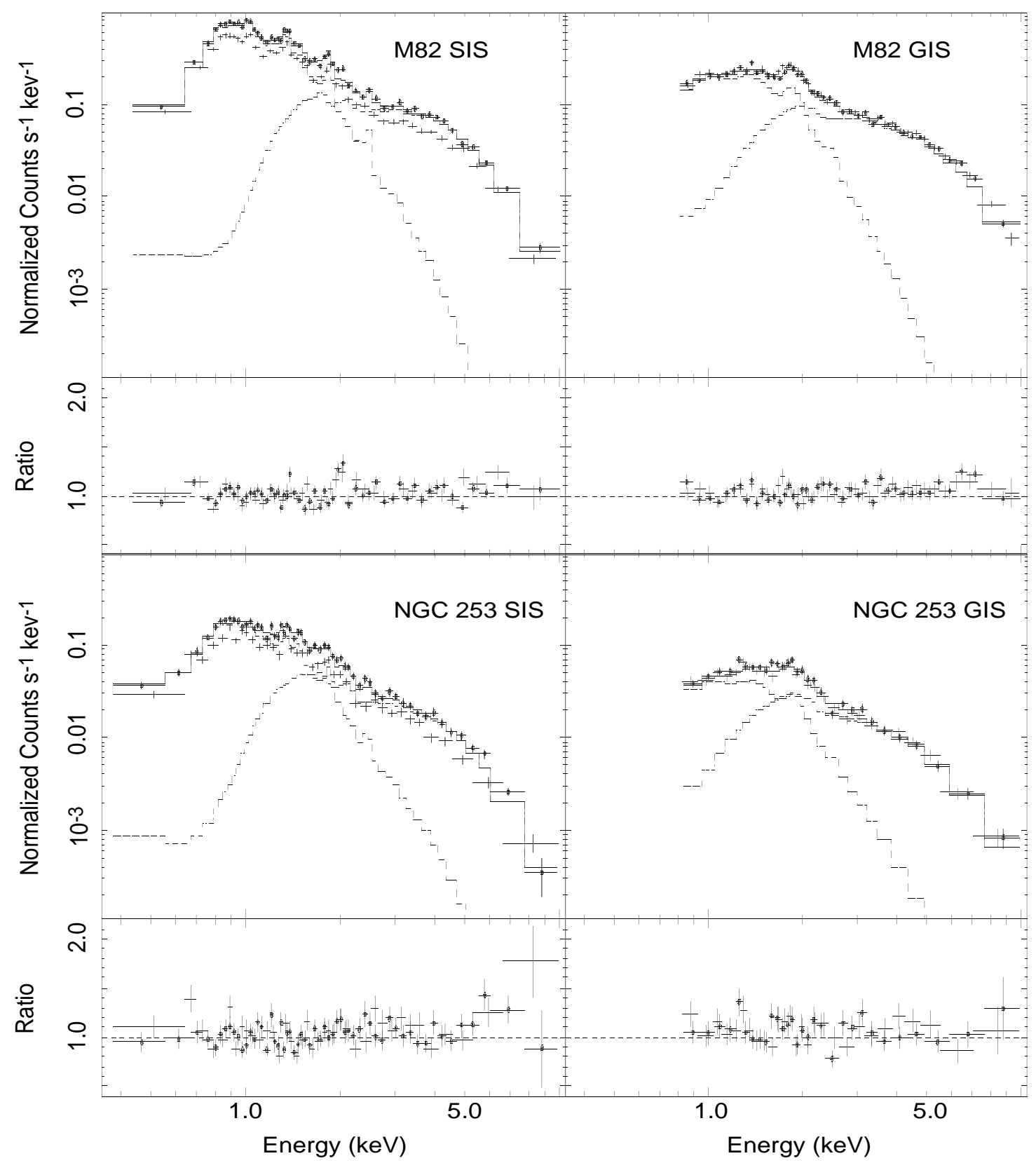

Fig. 2.- The best-fitting Raymond-Smith plus power-law model to the M82 and NGC 253 data. The top panels show the data with the model plotted as a solid line and the soft and hard components plotted separately as dashed lines, demonstrating that the hard component begins to dominate the spectra of both M82 and NGC 253 above $\sim 2 \mathrm{keV}$. The SIS and GIS data are shown separately for clarity, with the S0 and S2 points marked. The bottom panels show the ratio of the data to the models. As is evident in these figures, the SIS have a broader bandpass, with a low-energy cutoff of $\sim 0.4 \mathrm{keV}$, than the GIS, with a low-energy cutoff of $\sim 0.8 \mathrm{keV}$, while the GIS have more response at higher energies. 
not at a high statistical significance. The soft-component temperatures are similar $(\sim$ 0.6 and $0.8 \mathrm{keV}$, for M82 and NGC 253, respectively, insensitive to the choice of hardcomponent continuum) while the hard component appears to be somewhat harder in M82 $(\Gamma \sim 1.8$ or $\mathrm{kT} \sim 11 \mathrm{keV})$ than in NGC $253(\Gamma \sim 2.0$ or $\mathrm{kT} \sim 7 \mathrm{keV})$. If the hard component is thermal, the abundance (inferred from a Raymond-Smith model) is constrained to be significantly sub-solar $(<0.2$ and $<0.5$ in NGC 253 and M82, respectively), based primarily on the lack of a strong Fe-K line at 6.7-6.9 keV. In NGC 253 no (narrow) Fe$\mathrm{K}$ line emission is detected, and we set a $90 \%$ confidence upper-limit (for two additional parameters, the energy and normalization of the line) of $\sim 180 \mathrm{eV}$ for the EW of any line emission in the four-instrument Raymond-Smith plus power-law fit. In M82, the addition of a narrow line at $\sim 6.6 \mathrm{keV}$ with an $\mathrm{EW}$ of $\sim 100 \mathrm{eV}$ to the four-instrument RaymondSmith plus power-law fit reduces $\chi^{2}$ by $\sim 13$, significant at a level of $\sim 3 \sigma$ for two additional parameters. The Raymond-Smith plus power-law fits to the M82 and NGC 253 data are shown in Figure 2.

In M82 the soft-component abundances (for the S0-3 Raymond-Smith plus power-law fit) relative to solar were found to be: $\mathrm{N}<2.1$, $\mathrm{O}=0.18_{-0.14}^{+0.25}, \mathrm{Ne}=0.15_{-0.14}^{+0.20}, \mathrm{Mg}=0.22_{-0.11}^{+0.14}$, $\mathrm{Si}=0.35_{-0.11}^{+0.17}, \mathrm{~S}=0.60_{-0.37}^{+0.57}, \mathrm{Fe}=0.04_{-0.03}^{+0.02}$. The $\mathrm{Ar}, \mathrm{Ca}$, and $\mathrm{Ni}$ abundances were tied together (due to limited signal-to-noise) resulting in a mean value of $0.23_{-0.23}^{+0.36}$. Note that the Fe abundance inferred from the softcomponent model is determined solely based on Fe-L emission, not Fe-K, due to the dominance of the hard component above $\sim 2 \mathrm{keV}$. We note that these abundances were derived assuming that the soft component is entirely thermal. The poorer signal-to-noise of the NGC 253 data do not warrant allowing the elemental abundances to vary independently. $\mathrm{O}, \mathrm{Mg}, \mathrm{Si}$, and $\mathrm{S}$, which show strong line emission in Figure 1, were grouped together, and $\mathrm{Ne}$ and Fe-L, which are not resolved from each other, were grouped separately. The resulting abundances were $\mathrm{N}, \mathrm{Ar}, \mathrm{Ca}, \mathrm{Ni}<0.66 ; \mathrm{O}, \mathrm{Mg}$, $\mathrm{Si}, \mathrm{S}=0.27_{-0.14}^{+0.67}$ and $\mathrm{Ne}, \mathrm{Fe}=0.05_{-0.04}^{+0.15}$. As in M82, these results are suggestive of a depression of $\mathrm{Fe}$ abundance relative to $\mathrm{O}, \mathrm{Mg}$, $\mathrm{Si}$ and $\mathrm{S}$.

The somewhat high value of $\chi^{2}$ resulting from the SIS-only fits to the M82 data (see Table 1) suggests that a third component may be required. Relative minima in $\chi^{2}$ space are present in the M82 fits when the softcomponent temperature and $N_{H}$ are varied. This results in a highly assymmetric confidence interval for the temperature (see Table 1 ). In the case of $N_{H}$, the required difference in $\chi^{2}$ is reached before the onset of the new minima, giving the lower limit shown in Table 1, however the "true" lower limit on $N_{H}$ may be zero. This behavior also suggests the presence of an additional component, with a lower temperature than that of the soft component. The addition of a third thermal component to the double Raymond-Smith model substantially improves the M82 fits, resulting in temperatures of $0.35 \mathrm{keV}, 1.07 \mathrm{keV}$ and $12.4 \mathrm{keV}$ (see Tsuru et al., 1997 for a discussion of three-component Raymond-Smith and non-equilibrium plasma fits to the M82 spectra). Adding an additional thermal component to the double Raymond-Smith NGC 253 model reduced $\chi^{2}$ by only 8.3 for 2 additional degrees of freedom (kT and normalization, with $N_{H}$ fixed at the Galactic value and the abundances tied to the $\mathrm{T} \sim 10^{7} \mathrm{~K}$ component abundances), significant only at the $2.4 \sigma$ 
level. The temperature of the additional component was $\sim 0.14 \mathrm{keV}$ and the best-fitting model parameters of the other two components were similar to the two-component fit values in Table 1.

In Figure 3 we plot the abundances relative to $\mathrm{Fe}(\mathrm{x} / \mathrm{Fe})$ derived from various fits to the M82 data. In addition to the M82 fits in Table 1 and the triple Raymond-Smith model discussed above, these fits include substituting the Mewe-Kaastra-Liedahl plasma model ('mekal' in XSPEC) for the Raymond-Smith plasma model in the Raymond-Smith plus power law model, a power-law distribution of temperatures model, a double RaymondSmith plus power-law model, and including an additional (very steep) power-law in the triple Raymond-Smith model. While these fits result in abundances that are one to two orders of magnitude supersolar (indicating that the soft-component continuum is poorly constrained), Figure 3 shows that the relative abundances are somewhat more robust, with most varying by less than a factor of $\sim 5$, although the relative abundances of $\mathrm{Ar}, \mathrm{Ca}$ and $\mathrm{Ni}$ are poorly constrained. Note that these errors are still somewhat larger than the statistical errors discussed above, showing that systematic errors dominate. These systematic errors are most likley dominated by differences in the slope of the continuum across the line-emitting energies in each fit. A comparison of the Raymond-Smith and the Mewe-Kaastra-Liedahl models in XSPEC for a $0.6 \mathrm{keV}$, solar-abundance plasma shows that, with the exeption of an Oxygen line at $\sim 0.8 \mathrm{keV}$ present in the Raymond-Smith model but not in the mekal model, most of the differences between the two models are on the order of $30 \%$ or less, implying that uncertainties in atomic physics are likely to be much

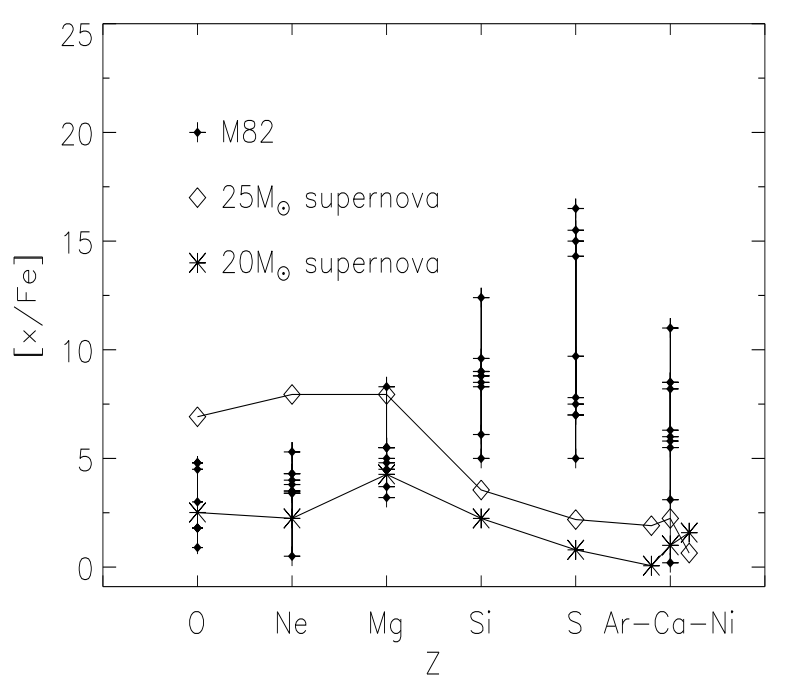

Fig. 3.- The abundances of various elements relative to Fe. The multiple points plotted for each element show the range of relative abundances derived from various models (see text). Note that the ranges shown are dominated by systematic errors which are much larger than the statistical errors (not explicitly shown), such as those for the fits given in Table 1. Also shown are the yields predicted for $20 M_{\odot}$ and $25 M_{\odot}$ supernovae, taken from Thielemann, Nomoto, \& Hashimoto (1996), normalized to Fe. 
less significant than the statistical errors in these data. Also shown in Figure 3 are the x/Fe ratios expected for 20 and $25 M_{\odot}$ supernova showing that even the direct emission of Type-II supernovae should not show such a large (relative) underabundance of Fe, particularly relative to $\mathrm{Si}$ and $\mathrm{S}$ ( Thielemann et al. 1996).

In Table 2 we give the observed fluxes and absorption-corrected luminosities (given for each component separately) for NGC 253 and M82 in the $0.5-2.0 \mathrm{keV}$ and $2-10 \mathrm{keV}$ bands, with the SIS values corrected for non-uniform spatial exposure. The fluxes inferred from the individual detectors are within $3-4 \%$ of the mean values, although systematic differences of $10-20 \%$ between the SIS and GIS values are often found in other sources. The uncertainty in $\mathrm{N}_{H}$ is significant in computing the unabsorbed luminosities, with the $90 \%$ confidence interval in $N_{H}^{\text {soft }}$ in NGC 253 corresponding to an uncertainty of $\sim 64 \%$ in intrinsic 0.5 $2.0 \mathrm{keV}$ luminosity. The $0.5-2.0 \mathrm{keV}$ PSPC fluxes for M82 and NGC 253 from 6' regions are $\sim 30 \%$ and $10 \%$ higher than the $A S C A$ fluxes, respectively, and lie within the scatter of the correlation of the $A S C A$ and $P S P C$ fluxes for 13 low-activity galaxies (including M82 and NGC 253) shown in Serlemitsos, Ptak \& Yaqoob (1996). Our 2-10 keV fluxes are $\sim 1 / 2$ the Ginga values (Ohashi et al. 1990, Tsuru et al. 1990) although any interpretation of this result must be viewed with caution given the large $\left(1^{\circ}\right.$ by $\left.2^{\circ}\right)$ Ginga FOV (however, the fact that the M82 observation was performed in a scanning mode reduces the uncertainty in flux).

\section{Discussion}

\subsection{Comparison with Superwind Mod- els}

While two and three-component models describe the data well, the true situation, particularly for the soft component below $\sim 2$ $\mathrm{keV}$, is likely to be a distribution of temperatures. For example, the ROSAT PSPC data from both M82 and NGC 253 suggest temperatures of $\sim 10^{7} \mathrm{~K}$ in their disk and nuclear regions, with the temperature decreasing with radius out in the halos (i.e., beyond $\sim 1 \mathrm{kpc}$ ), as discussed in Strickland, Ponman, \& Stevens (1996) and Dahlem, Heckman \& Weaver (1996). In NGC 253, the soft $(<2$ $\mathrm{keV}$ ) component is apparently dominated by a disk and nuclear thermal component with $\mathrm{kT} \sim 0.8 \mathrm{keV}$. In M82, on the other hand, the soft component appears to have approximately equal contributions from $\sim 1.1 \mathrm{keV}$ gas in the nuclear and disk regions and $\sim$ $0.4 \mathrm{keV}$ gas in the halo, which is fit reasonably well by a $0.8 \mathrm{keV}$ thermal model. The luminosities, pressures, masses and energetics implied by the best-fitting soft-component model parameters are consistent with recent models of starburst-driven winds (Tomisaka \& Bregman 1993; Suchkov et al. 1996), although the details of this kind of analysis are sensitive to assumptions concerning the volume, filling factor, and possibly the outflow velocity of the gas associated with each component. For example, the outflow velocity of the X-ray emitting gas in M82 may be as high as $1000-3000 \mathrm{~km} \mathrm{~s}^{-1}$ (Seaquist \& Odegard 1991) although the outflow velocity of molecular gas in M82 is estimated to be less than $500 \mathrm{~km} \mathrm{~s}^{-1}$ (Nakai et al. 1987). To estimate the contribution of SN to the observed thermal flux we assume that the ISM heating is dominated by type-II SN, and that each supernova ejects $\sim 10 M_{\odot}$ with $10^{51}$ ergs 
Table 2: ASCA Fluxes and Luminosities for M82 and NGC 253

\begin{tabular}{|c|c|c|c|c|c|c|c|}
\hline \multirow[t]{3}{*}{ Galaxy } & \multirow[t]{3}{*}{ Energy Range } & \multirow[t]{3}{*}{$\begin{array}{c}\text { Observed Flux } \\
\left(10^{-12} \operatorname{ergs~s}^{-1} \mathrm{~cm}^{-2}\right)\end{array}$} & \multicolumn{5}{|c|}{$\begin{array}{l}\text { Absorption-Corrected Luminosities } \\
\qquad\left(10^{40} \mathrm{ergs} \mathrm{s}^{-1}\right)\end{array}$} \\
\hline & & & \multicolumn{2}{|c|}{ Two-comp. Fit ${ }^{\dagger}$} & \multicolumn{3}{|c|}{ Three-comp. Fit* } \\
\hline & & & Soft & Hard & Soft & Medium & Hard \\
\hline M82 & $0.5-2.0 \mathrm{keV}$ & 9.4 & 2.7 & 2.1 & 3.2 & 2.5 & 1.4 \\
\hline M82 & $2.0-10.0 \mathrm{keV}$ & 20 & 0.2 & 3.5 & 0.0 & 0.6 & 3.4 \\
\hline NGC 253 & $0.5-2.0 \mathrm{keV}$ & 2.8 & 0.23 & 0.33 & 0.02 & 0.26 & 0.19 \\
\hline NGC 253 & $2.0-10.0 \mathrm{keV}$ & 4.7 & 0.03 & 0.37 & 0.00 & 0.03 & 0.35 \\
\hline
\end{tabular}

All values are averages from S0-3 fits [M82 (NGC 253) fluxes from individual detectors are within $3 \%(4 \%)$ of the mean].

Observed fluxes are inferred from the best-fitting two-component model (three-component models give similar results).

$\dagger$ Power law (hard) plus Raymond-Smith (soft) model

* Triple Raymond-Smith model

of kinetic energy (c.f., Suchkov et al. 1996). With these assumptions, independent of the assumed volume $V$ and filling factor,

$$
\begin{aligned}
\frac{M_{S N}}{M_{\text {total }}} & \sim \frac{\left(\frac{3}{2} n V k T+\frac{1}{2} m_{H} n V v_{1000}^{2}\right) M_{10}}{10^{51} m_{H} n V} \\
& \sim \frac{\frac{3}{2}\left(k T+m_{H} v_{1000}^{2}\right) M_{10}}{10^{51} m_{H}}
\end{aligned}
$$

where $M_{S N}$ is the mass of gas ejected by SN (i.e., individual SN and the superwind), $M_{\text {total }}$ is the total X-ray emitting mass, $M_{10}$ is the mass ejected by a typical SN in units of $10 M_{\odot}$ and $v_{1000}$ is the outflow velocity in units of $1000 \mathrm{~km} \mathrm{~s}^{-1}$. We estimate (with $M_{10}=1$ and $\left.v_{1000}=1\right)$ that $\frac{M_{S N}}{M_{\text {total }}} \sim 12-14 \%$ for the $1.1 \mathrm{keV}$ and $0.8 \mathrm{keV}$ gas (for M82 and NGC 253 , respectively) of the soft-component emission. $M_{10}$ and $v_{1000}$ are likely to be somewhat smaller than $\sim 3$, so this implies that a significant amount of the emission is due to ambient ISM, possibly "swept-up" by the superwind, as suggested by Suchkov et al. (1996). The low absolute abundances implied by some of the models of the soft component may therefore be indicative of low abundances in the (ambient and swept-up) disk ISM. This calculation is not appropriate for the $\sim 0.4 \mathrm{keV}$ halo gas in M82 since energy dissipation is likely to significant out in the halo. The unusually high values of $\mathrm{x} / \mathrm{Fe}$ shown in Figure 3 indicate that the Fe abundance is "suppressed", particularly relative to $\mathrm{S}$ and Si. The high IR luminosities of M82 and NGC 253 suggest a significant dust distribution in the disk and halo ISM of M82 and NGC 253 (Telesco 1988). Since Fe is depleted in warm ISM clouds to a larger extent than $\mathrm{S}$ and $\mathrm{Si}$ (c.f., Cowie \& Songaila 1986), dust depletion may account for some of the Fe deficiency, resulting in $\mathrm{Si} / \mathrm{Fe}$ and $\mathrm{S} / \mathrm{Fe}$ ratios $\sim 17 \%$ and $130 \%$ (respectively) higher than in the unde- 
pleted case 3 .

Repeating the "swept-up mass" calculation described above for the hard component, we find that, if thermal, 25-75\% (NGC 253) and $40-100 \%$ (M82) of the hard component originates in superwind emission and not the ambient ISM. The high absorption $\left(N_{H} \sim\right.$ $10^{22} \mathrm{~cm}^{-2}$ ) derived from fits to the hard components (see Table 1) also suggests that the hard component originates in compact and/or nuclear regions (note that radio observations of the nuclear regions of both M82 and NGC 253 suggest the presence of molecular gas with similar column densities; Nakai et al. 1986; Paglione, Tosaki, \& Jackson 1996). However, the inconsistency of the low abundances inferred from the hard-component fits with the high metallicity expected in supernova ejecta and solar abundances expected in the nuclear HII regions of M82 and NGC 253 (c.f., Carral et al. 1993; Lord et al. 1996) suggest that the hard components may not be dominated by (nuclear) superwind emission. Dust depletion may again be important although dust destruction is likely to be significant in nuclear starburst regions over the $\sim 10^{7-8}$ year lifetime of the starburst ( Calzetti, Kinney \& Storchi-Bergmann 1996). It is therefore likely that gas not in thermal equilibrium or non-thermal emission contribute significantly to the hard components. The former case would correspond to $n_{0}<$ $0.5-1.5 \times 10^{-3}\left(v_{1000} / r_{k p c}\right) \mathrm{cm}^{-3}$, where $r_{k p c}$ is the hard-component emitting region size in $\mathrm{kpc}, v_{1000}$ is the velocity of the ejecta (as defined above), and $n_{0}$ is the pre-shock ambient density (c.f., Tomisaka \& Bregman 1993;

\footnotetext{
${ }^{3}$ The dust depletion given in table 3 of Cowie \& Songalia was scaled to the $\sim 50 \%$ depletion of Si estimated by Lord et al. (1996) for the nuclear HII regions in M82 in this calculation.
}

Hamilton, Sarazin, \& Chevalier 1983). Even if $v_{1000}$ is as high as 3 , such a low value of $n_{0}$ is unlikely in the nuclear regions of M82 and NGC 253 (Carral et al. 1993; Lord et al. 1996), suggesting that non-thermal equilibrium ionization is not important unless the direct emission of $\mathrm{SN}$ is significant. Using the radio surface brightness - radius relationship for SN in Huang et al. (1993) and the Xray luminosities of SN1986J and Cas A as a guide, we expect the X-ray luminosity of SN in M82 to be $\sim 10^{37-38} \mathrm{ergs} \mathrm{s}^{-1}$, too low to account for more than a few percent of the hard component, while in NGC 253 some of the bright extranuclear sources may be supernovae contributing to the hard component. Future work will examine the possible thermal contribution to the hard component in more detail, but it appears likely that the hard components in M82 and NGC 253 are dominated by non-thermal emission.

\subsection{The Nature of the Non-thermal Emission}

The two most likely candidates for nonthermal emission are accretion-driven point sources (e.g., X-ray binaries and AGN) and inverse-Compton (IC) scattering of (nuclear) IR flux by relativistic electrons. The IC component may be significant (estimated by Schaaf et al. 1991 to be $L_{1.4-8.9 \mathrm{keV}} \sim 1.5 \times$ $10^{40} \mathrm{ergs} \mathrm{s}^{-1}$ or $\sim 50 \%$ of the hard component in M82), although the likely high ratio of proton to electron energies (i.e., 100; Seaquist \& Odegard 1991) would significantly reduce the contribution of this component. On the other hand, the point sources in M82 and NGC 253 may account for much of the hard component. In M82, a nuclear source has varied from $\sim 0.04-0.06$ counts $\mathrm{s}^{-1}$ to $\sim$ 0.02 counts s $^{-1}$ between the 1991 ( Collura et 


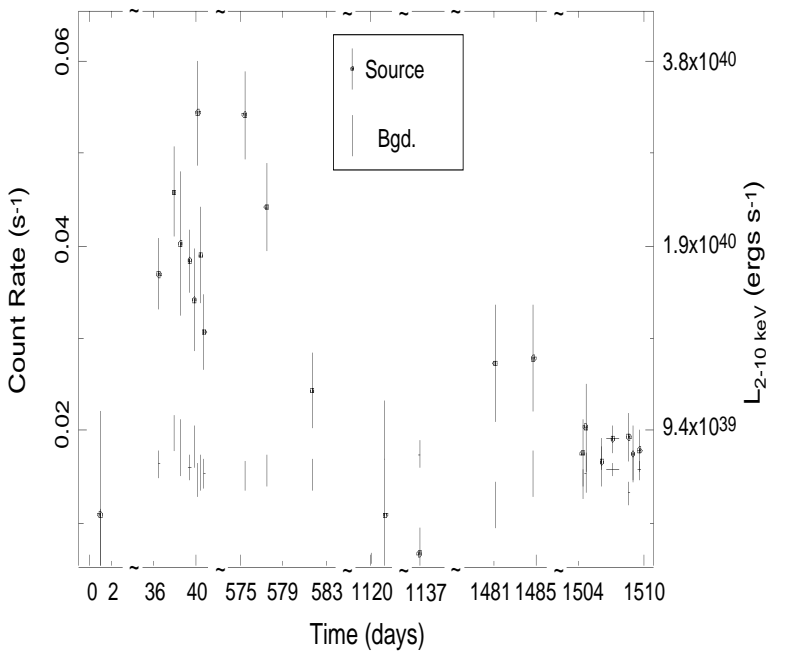

Fig. 4.- The ROSAT HRI backgroundsubtracted light curve for the nuclear source in M82 (c.f., Figure 2 of Collura et al. 1994), including recent AO-5 data. The source counts were extracted from a 10" region centered on the source and the background was determined from a 15"-20" annulus centered on the source (here the background is completely dominated by nuclear emission in M82). Also shown is the corresponding absorption-corrected 2-10 keV luminosity assuming a spectrum similar to the hard component inferred from the $A S C A$ data (i.e., $\Gamma \sim 1.8$ and $N_{H} \sim 10^{22} \mathrm{~cm}^{-2}$ ). Evidently, this source could account for a considerable fraction of the intrinsic $2-10 \mathrm{keV}$ luminosity of M82 $\left(L_{X} \sim 3.4 \times 10^{40} \mathrm{ergs} \mathrm{s}^{-1}\right)$ observed by $A S C A$ (on day 755 in this figure). al. 1994) and 1995 ROSAT HRI observations, corresponding to an intrinsic $2-10 \mathrm{keV}$ luminosity of $1-4 \times 10^{40} \mathrm{ergs} \mathrm{s}^{-1}$ or $\sim 30 \%-100 \%$ of the hard-component luminosity assuming this source has a spectrum similar to the powerlaw model fit to the hard component (see Table 1 and Figure 4). In NGC 253, four strong extranuclear point sources $(\sim 2-4$ ' from the nucleus) are responsible for $\sim 20 \%$ of the 0.5 $2.0 \mathrm{keV}$ flux in the ROSAT PSPC observation. As shown in Figure 5 (plate 1), the point sources in NGC 253 have varied between the Einstein and ROSAT observations and are clearly prominent above $2 \mathrm{keV}$ in the $A S C A$ S0 image. We extracted composite SIS spectra for the two brightest extranuclear sources (sources R1 and R5 in figure 5), using source region sizes of 45 " to minimize the "contamination" from diffuse gas. Fitting these spectra with the Raymond-Smith plus power-law model (see Table 1) resulted in fit parameters consistent with those listed in Table 1 for the entire galaxy (e.g., $N_{H} \sim 2.1_{-1.9}^{+7.4} \times 10^{22} \mathrm{~cm}^{-2}$ and $\Gamma \sim 2.3_{-1.0}^{+1.7}$ ). These fits suggest that sources R1 and 5 account for $\sim 25 \%$ of the observed 2-10 keV flux from NGC 253. While it is possible that some of the point sources in NGC 253 are associated with SN (see above), some are likely to be X-ray binaries given the lack of optical counterparts (i.e., Fabbiano \& Trinchieri 1984). The Eddington luminosities of these sources suggest masses of at least 3-20 $M_{\odot}$ implying that they are blackhole candidates, which also have spectra consistent with the hard components observed in M82 and NGC 253 (Tanaka 1989). The spectra of M82 and NGC 253 are also very similar to the spectra of low-luminosity AGN and LINERs (Serlemitsos, Ptak and Yaqoob 1996) which generally can be described by a Raymond-Smith plus power-law model. The power-law slopes obtained from our fits $(\Gamma \sim 1.8-2.2)$ may 
suggest a common physical origin, such as an accretion-driven component. It is likely that some of the point sources in M82 and NGC 253 have masses $\gg 10 M_{\odot}$ since it would be unphysical for all of these sources to be emitting at their Eddington luminosities most of the time, if they are powered by accretion. The discovery of (long-term) X-ray variability in "starburst" galaxy NGC 3628 demonstrates that the X-ray flux from NGC 3628 is dominated by a point source, most likely a massive blackhole candidate or a micro-AGN ( Dahlem, Heckman, Fabbiano 1995; Yaqoob et al. 1995). These considerations suggest the possibility that starburst galaxies such as M82 and NGC 253 may harbor blackhole candidates, with masses significantly higher than the masses of blackhole candidates in our galaxy.

The authors wish to thank all of the members of $A S C A$ team who have made this work possible. AP wishes to acknowledge the support of the NASA Graduate Student Research Program. This research has made use of the NASA's Astrophysics Data System Abstract Service and the NASA/IPCA Extragalactic Database (NED) which is operated by the Jet Propulsion Laboratory, Caltech, under contract with NASA. This work will be included in a dissertation to be submitted to the Graduate School, University of Maryland, in partial fulfillment of the requirements for the Ph.D. degree in Physics.

\section{Appendix: Computation of the $A S C A$ X-Ray Telescope (XRT) Response for Extended Sources}

As stated in the text, the X-ray emission in both M82 and NGC 253 is extended while the telescope response was computed assum- ing it is point-like. In this appendix we show that properly taking the extended nature of the sources into account does not significantly impact the results of this paper. In X-ray spectral fitting, the XRT response is taken into account by the creation of a table of effective area as a function of energy, which is then used to convert the observed counting rate in each spectral bin to a flux. This table, hereafter referred to as an ARF (ancillary response file), also applies a correction for the counts scattered out of the source region (6' circular regions were used for both the SIS and GIS in this paper).

\section{The $A S C A$ XRT Response for a Point Source}

First, we describe the "standard" procedure used for the creation of an ARF appropriate for a point source. The telescope point-spread function (PSF) is summed over the source region to determine percentage of source photons contained in the source region. The PSF sum is then multiplied by the telescope effective area. The PSF and effective area are functions of both energy and the off-axis angle of the source. Both the PSF and effective area are interpolated from values contained in calibration look-up tables, computed using a Monte-Carlo procedure based on a raytracing program. Although the XRT PSF has a cross-like shape, the calibration tables contain azimuthally-averaged values, an approach that is usually a good approximation as long as most of the source counts lie in a symmetric (and preferably circular) region. The mirrors contain a heat shield, made up of a thin plastic film, to protect against temperature gradients, and the computed effective area is reduced to take photon losses through the shield into account. The gas cell 


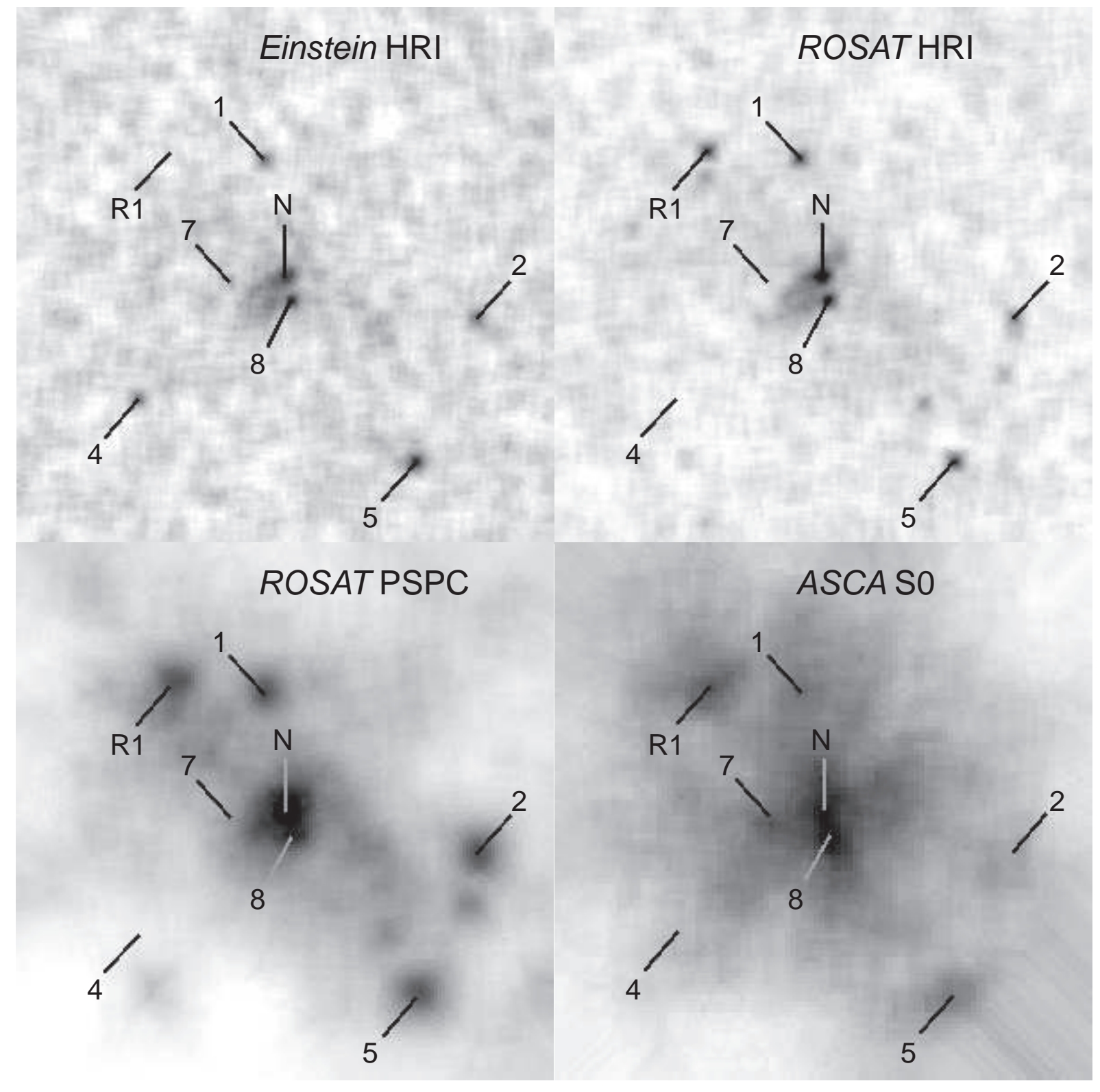

Fig. 5.- Einstein HRI (0.2-4.0 keV, 7/5-9/79; top left), ROSAT HRI (0.1-2.2 keV; 6/7/92; top right), ROSAT PSPC (0.5-2.0 keV; 12/6/91-5/6/92; bottom left), and ASCA S0 (2-10 keV; $5 / 25 / 93$; bottom right) images of NGC 253 . Each image is $10^{\prime}$ by $10^{\prime}$ and has been adaptively smoothed to produce a SNR of $\gtrsim 5$ at each pixel (without background subtraction). The sources discussed in Fabbiano \& Trinchieri (1984) are marked (sources 3 and 6 lie outside of the FOV of these images) along with the X-ray nucleus ('N') and the "new" ROSAT source ('R1'). 
of the GIS contains a Beryllium window held in place by a supporting grid, and transmission through the grid and Be must also be taken into account. Note that the thicknesses of both the grid and Be vary across the GIS detectors. Also, unlike the SIS, the GIS introduce a broadening of the PSF. The instrumental PSF of the GIS is approximately a Gaussian whose FWHM $\sim 0.5 \sqrt{\frac{5.9 \mathrm{keV}}{E}}$ arcminutes, with the addition of a "tail" at energies $\gtrsim 4 \mathrm{keV}$. Accordingly, the XRT PSF is modified by the grid and Be transmission and then convolved with the GIS PSF, which is modeled by the sum of two-dimensional Gaussians. Hereafter, we refer to ARFs computed with this approach as standard ARFs.

\section{Computation of the $A S C A$ Response to an Extended Source}

There are two ways in which standard ARFs may be inappropriate for an extended source. First, more source counts would be scattered out of the source region than is the case for a point source. Not taking this into account would result in an underestimate of the source flux. Second, by definition, an extended source subtends a distribution of offaxis angles, so determining the XRT effective area and PSF for a single off-axis angle may be incorrect. Because both the effective area and PSF have an energy dependence, the spectral results may be affected as well as the flux estimates. Because the X-ray emission from M82 and NGC 253 is relatively compact and weak, it is likely that statistical errors will dominate that the effects described above, with the possible exception that the fluxes may be underestimated. To check this in detail, we adopted a Monte-Carlo approach. As is the case in the point-source procedure, we divided the bandpasses of the SIS and GIS into the same number of energy bins as is used in the spectral fitting, namely 1180 in the case of the SIS and 201 in the case of the GIS. For each bin at least 10000 photons were raytraced through the mirror (the raytracing code was developed by Peter Serlemitsos during the design of the $A S C A$ mirrors, and, when azimuthally-averaged, is in good agreement with the look-up tables described above and the radial profile of an $A S C A \mathrm{PV}$ phase observation of $3 \mathrm{C} 273$ ). The position (on the sky) of each photon was chosen at random from ROSAT PSPC images of M82 and NGC 253, weighted by the intensity at each pixel. This approach results in photons incident on the XRT with a distribution in off-axis angles consistent with those expected from the PSPC images. A 12' by 12' image was used for M82 and a 10' by 10' image was used for NGC 253. The bandpass of each image was limited to $\sim 0.5-2.0 \mathrm{keV}$, and the background was not subtracted, in order to conservatively overestimate the source extent (note that the source extent is also overestimated since the ROSAT PSPC PSF is not removed with this procedure, although the ROSAT PSF FWHM is $\sim 10^{\prime \prime}$, somewhat smaller than the $A S C A$ XRT PSF FWHM of $\sim 50^{\prime \prime}$ ).

In the case of the GIS, the instrumental PSF was introduced by adding "noise" (i.e., Gaussian random deviates) to the raytraced photon positions. The standard deviations of the deviates were computed with the same algorithm used in the standard ARF procedure. Also in the case of the GIS, photons were rejected to account for the grid and Be transmission (i.e., a photon was rejected if a uniform deviate exceeded the product of the Be and grid transmission at the photon's position). The percentage of photons "detected" 
in the source region, reduced by the thermal shield transmission (as is done in the point source case), was then multiplied by the geometrical area of the mirror to derive an effective area. Hereafter, we refer to ARFs computed with this procedure as "raytraced" (although, as stated above, the "standard" ARF procedure also indirectly involves raytracing, in the computation of the look-up tables).

Initially, ARFs were raytraced assuming that M82 and NGC 253 are point sources for comparison with the standard ARFs used in the fits given in the text. The ratio of the raytraced ARFs to the standards ARFs, shown in the top panel of figure 6 for the case of the M82 S0 and S2 ARFs, gives the systematic differences between the two procedures described above. The largest source of discrepancy between the two methods is due to differences in the raytracing code used here and the raytracing used to produce the lookup tables, particularly in the choice of optical constants for gold (the reflecting material used in the XRT). Other sources of discrepancy are inherent to the procedures employed, i.e., the XRT PSF is not azimuthally-averaged in the raytraced case. The top panel of figure 6 shows that, with the exception of a sharp peak around the $\mathrm{Au} \mathrm{M}$ edge at $2.2 \mathrm{keV}$, the two procedures are in agreement below $4 \mathrm{keV}$ to within $10 \%$. Above $4 \mathrm{keV}$ there is a broad "hump" in the ratio plot which peaks at a level of $\sim 20 \%$. These are issues which are currently being resolved by the instrument teams and will be reported in a future issue of ASCANews. For comparison, the statistical errors in the ASCA data below $4 \mathrm{keV}$ are typically on the order of $6 \%$ in the case of M82 and $10 \%$ in the case of NGC 253. As is evident in Figure 2, the statistical errors become somewhat larger above $4 \mathrm{keV}$ since both

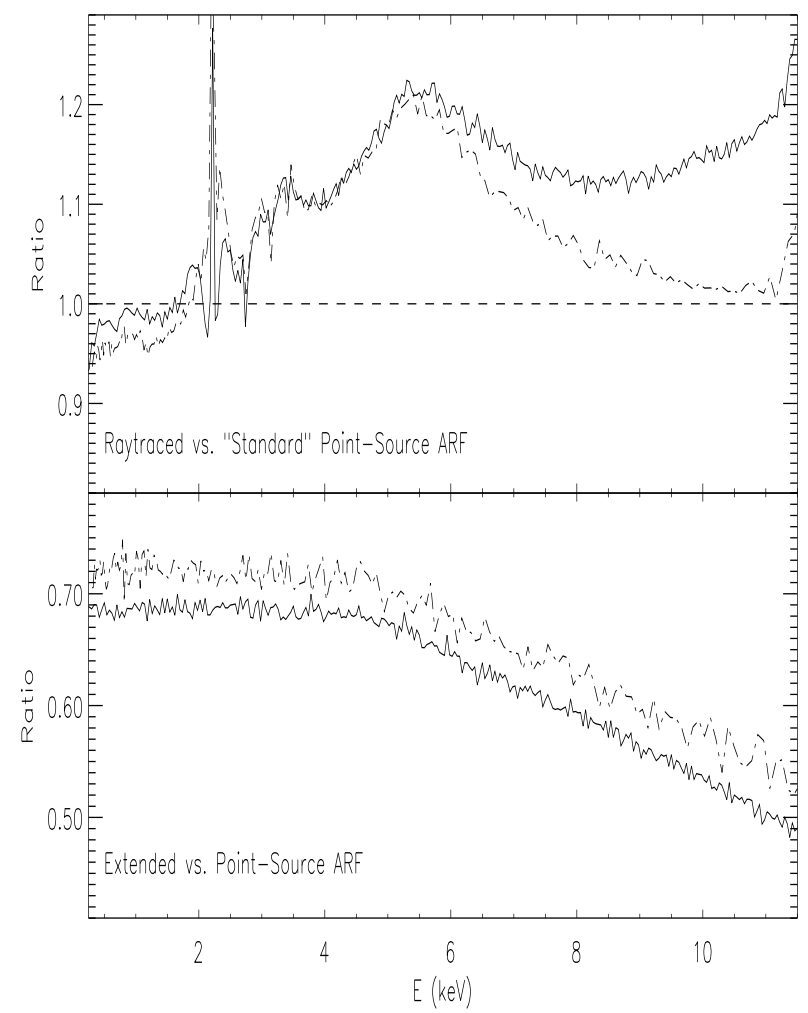

Fig. 6.- (top) The ratio of ARFs for a point source computed using the raytracing algorithm described in the text and the "standard" procedure using look-up tables for the XRT effective area and PSF. The ratio for ARFs appropriate for the S0 (solid line) and G2 (dashed-line) M82 data are shown. The largest source of discrepancy between the raytraced and "standard" ARFs arises from differences in the raytracing code used in this analysis and the raytracing used to produce the look-up tables. (bottom) The ratio of the ARFs computed assuming that M82 is extended and assuming that M82 is a point source in the case of S0 (solid-line) and S2 (dashed line). Here, both the point-source and extended-source ARFs were raytraced to remove the discrepancies between the raytraced and "standard" procedures (shown in the top panel). 
the XRT and instrumental responses and the source fluxes drop off rapidly above $4 \mathrm{keV}$, particularly in the case of NGC 253 which has a steeper spectrum than M82.

\section{Implications for M82 and NGC 253}

The bottom panel of figure 6 shows that ratio of ARFs produced with the raytracing procedure for an extended source (with photons selected from the PSPC image, as described above) and a point-source (raytraced ARFs were computed in both cases to remove the discrepancy between the raytraced and standard ARFs) in the case of the M82 SIS0 (solid line) and GIS2 (dashed line) ARFs. Note that the extended nature of the source only impacts the overall normalization below $\sim 4 \mathrm{keV}$, and results in a gradual drop off in net effective area above $4 \mathrm{keV}$ (a result of the larger energy dependence of the XRT PSF and effective area above $4 \mathrm{keV}$ ). Note that this drop in effective area partially offsets the "hump" above $4 \mathrm{keV}$ noted above. Indeed, in the case of NGC 253, repeating the four-instrument Raymond-Smith plus power-law fit with the extended source ARFs results in best-fitting parameters that are essentially identical to those discussed in the text where the standard ARFs were used (i.e., the statistical errors dominate any systematic errors). When the double RaymondSmith fit to the M82 data is repeated with the extended-source ARFs, the soft component parameters do not change significantly, although the inferred $0.5-2.0 \mathrm{keV}$ flux is increased by $\sim 40 \%$. Because our procedure deliberately overestimates the source extent, this is a conservative upper limit to the 0.5$2.0 \mathrm{keV}$ flux from M82. On the other hand, the hard component spectral parameters are modified slightly when extended-source ARFs are used, with the best-fitting $N_{H}$ decreasing by $\sim 5 \times 10^{21} \mathrm{~cm}^{-2}$, the temperature increasing by $\sim 3 \mathrm{keV}$ and the abundance increasing from $\sim 0.2$ solar to $\sim 0.3$ solar. Nevertheless, these parameters are still well within the statistical errors quoted in the text. As with the soft component, the extended-source fits suggest that the 2-10 keV flux, dominated by the hard component, may be underestimated by $\sim 27 \%$. However, Tsuru et al. (1997) report that the flux associated with the hard component in M82 is not resolved by $A S C A$. Recomputing the extended-source ARFs by selecting the soft-component photons from the PSPC image and assuming that the hard component is point-like eliminates the changes in the hard-component fit parameters, and results in a $2-10 \mathrm{keV}$ flux $\sim 7 \%$ lower than that inferred from the standard ARF fits (this is not surprising since, as shown in the top panel of Figure 6 , the raytraced point-source ARF gives a larger effective area at high energies than the standard ARFs). Thus, the only parameter significantly affected by properly computing the response of $A S C A$ to an extended source is the $0.5-2.0 \mathrm{keV}$ flux inferred for M82, which does not impact any of the conclusions of this paper.

\section{REFERENCES}

Bhattacharya, D., et al. 1994, ApJ, 437, 173

Bregman, J., Schulman, E., \& Tomisaka, K. 1995, ApJ, 439, 155

Calzetti, D., Kinney, A., \& Storchi-Bergmann, T. 1996, ApJ, 458, 132

Carral, P., Hollenbach, D., Lord, S., Colgan, S., Haas, M., Rubin, R., \& Erickson, E. 1994, ApJ, 423, 223

Carilli, C., Holdaway, M., Ho, P., \& De Pree, C. 1992, ApJ, 399, L59 
Collura, A., Reale, F., Schulman, E., \& Bregman, J. 1994, ApJ, 420, L63

Cowie, L. \& Songaila, A. 1986, ARA\&A, 24, 499

Dahlem, M., Heckman, T., \& Weaver, K. 1997, ApJ, in press

Dahlem, M., Heckman, T., \& Fabbiano, G. 1995, ApJ, 442, L49

Davidge, T. \& Pritchet, C. 1990, AJ, 100, 102

Fabbiano, G. 1988, ApJ, 330, 672

Fabbiano, G. \& Trinchieri, G. 1984, ApJ, 286, 491

Freedman, W. et al. 1994, ApJ, 427, 628

Hamilton, A., Sarazin, C., \& Chevalier, R. 1983, ApJS, 51, 115

Heckman, T., Armus, L., \& Miley, G. 1990, ApJS, 74, 833

Huang, Z., Thuan, T., Chevalier, R., Condon, J., \& Yin, Q. 1994, ApJ, 424, 114

Lord, S., Hollenbach, D., Haas, M., Rubin, R., Colgan, S., \& Erickson, E. 1996, ApJ, 465, 703

Mewe, R., Gronenschild, E., \& Van Den Oord, G. 1985, A\&AS, 62, 197

Moran, E. \& Lehnert, M. 1997, ApJ, in press

Nakai, N. et al. 1987, PASJ, 39, 685

Ohashi, T., Makishima, K., Tsuru, T., Takano, S., Koyama, K, \& Stewart, G. 1990, ApJ, 365,180

Paglione, T., Tosaki, T., \& Jackson, J. 1996, ApJ, 454, L117

Petre, R. 1993, in The Nearest Active Galaxies, eds. J. Beckman, L. Colina, and $\mathrm{H}$. Netzer, 117

Rieke, G., Lebofsky, M., Thompson, R., Low, F., \& Schneider, D. 1980, ApJ, 238, 24
Schaaf, R., Pietsch, W., Biermann, P., Kronberg P., \& Schmutzler, T. 1989, ApJ, 336, 722

Seaquist, E. \& Odegard, N. 1991, ApJ, 369, 320

Serlemitsos, P., Ptak, A., \& Yaqoob, T. 1996, in The Physics of LINERs in View of Recent Observations, eds., M. Eracleous, A. Koratkar, C. Leitherer, and L. Ho, 70

Stark, A. A., Gammie, C. F., Wilson, R. W., Bally, J., Linke, R., Heiles, C., \& Hurwitz,M. 1992, ApJS, 79, 77

Strickland, D., Ponman, T., \& Stevens, I. 1997, å, in press

Suchkov, A., Berman, V., Heckman, T., \& Balsara, D. 1996, ApJ, 463, 528

Yaqoob, T., Serlemitsos, P., Ptak, A., Mushotzky, R., Kunieda, H. \& Terashima, Y. 1995, ApJ, 455, 508

Tanaka, Y. 1989 in 23rd ESLAB Symp. on Two Topics in X-ray Astronomy, eds. J. Hunt and B. Battrick, 3

Tanaka, Y., Inoue, H., \& S. Holt 1994, PASJ, 46, L37

Telesco, C. 1988, ARA\&A, 26, 343

Thielemann, F., Nomoto, K., \& Hashimoto, M. 1996, ApJ, 460, 408

Tomisaka, K. \& Bregman, J. 1993, PASJ, 45, 513

Tsuru, T., et al. 1997, PASJ, submitted

Tsuru, T., Ohashi, T., Makishima, K., Mihara, T., \& Kondo, H. 1990, PASJ, 42, L75

Watson, M., Stanger, V., \& Griffiths, R. 1984, ApJ, 286, 144

This 2-column preprint was prepared with the AAS LATEX macros v4.0. 